INPLASY

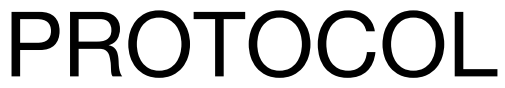

To cite: Manosroi et al.

Glycemic Abnormalities in

Primary Aldosteronism: A

systematic review and meta-

analysis. Inplasy protocol

202220004. doi:

10.37766/inplasy2022.2.0004

Received: 03 February 2022

Published: 04 February 2022

Corresponding author:

Worapaka Manosroi

worapaka.m@gmail.com

Author Affiliation:

Faculty of Medicine, Chiang

Mai University.

Support: No funding support.

Review Stage at time of this submission: Piloting of the study selection process.

Conflicts of interest:

None declared.

\section{Glycemic Abnormalities in Primary Aldosteronism: A systematic review and meta-analysis}

Manosroi, W1; Atthakomol, $\mathrm{P}^{2}$.

Review question / Objective: Is there a differences of prevalence glycemic abnormalities between primary aldosteronism (PA) and essential hypertension (EH) patients? Condition being studied: The present systematic review and meta-analysis aimed to systemically update findings regarding differences in the incidence of glycemic abnormalities and other glycemic abnormalities in PA and EH patients.

Eligibility criteria: 1) observational and comparative studies of PA and EH patients age over 18 years; 2) studies containing at least one of the following: data on prevalence of abnormal glucose metabolism and 3) These data should be compared between PA and EH patients and be presented as outcomes of interest, baseline characteristics or baseline investigations.

INPLASY registration number: This protocol was registered with the International Platform of Registered Systematic Review and Meta-Analysis Protocols (INPLASY) on 04 February 2022 and was last updated on 04 February 2022 (registration number INPLASY202220004).

\section{INTRODUCTION}

Review question / Objective: Is there a differences of prevalence glycemic abnormalities between primary aldosteronism (PA) and essential hypertension (EH) patients?

Rationale: A 2014 meta-analysis conducted by Chen et al. reported that abnormal glucose metabolism was more prevalent in
PA than in EH patients. However, that study contained multiple methodological errors. The association of glycemic abnormalities between primary aldosteronism (PA) patients and essential hypertension (EH) patients is still inconclusive.

Condition being studied: The present systematic review and meta-analysis aimed to systemically update findings regarding differences in the incidence of glycemic 
abnormalities and other glycemic abnormalities in PA and EH patients.

\section{METHODS}

Search strategy: Four databases, PubMed/ MEDLINE, Scopus, Cochrane and Web of Science, will be searched. The keywords included were "hyperaldosteronism" AND "glucose OR insulin OR diabetes mellitus OR insulin resistance OR glucose metabolism OR glucose tolerance OR HOMA OR impaired glucose". References from the included studies, other relevant publications and non-included reviews will be identified and included as additional studies for the initial screening.

Participant or population: Patients with primary aldosteronism (PA).

Intervention: N/A.

Comparator: Essential hypertension patients.

Study designs to be included: Observational study (cohort, case-control, crossectional).

Eligibility criteria: 1) observational and comparative studies of PA and EH patients age over 18 years; 2) studies containing at least one of the following: data on prevalence of abnormal glucose metabolism and 3) These data should be compared between PA and EH patients and be presented as outcomes of interest, baseline characteristics or baseline investigations.

Information sources: Electronic databases only.

Main outcome(s): Outcomes of interests including percentage of abnormal glucose metabolism and alterations of glucose metabolic parameters between PA and EH patients.

Additional outcome(s): Additional outcome of interests including percentage of abnormal glucose metabolism including DM, IFG and IGT; glucose metabolic parameters including mean and SD of FBG, HbA1c, 2-hr OGTT, HOMA-IR, HOMA-B, AUC of glucose, AUC of insulin and QUICKI for each group of PA and EH patients.

Data management: Rayyan, a web-based program, was employed for duplicate removal and initial screening of abstracts and titles.

Quality assessment / Risk of bias analysis: Joanna Briggs Institute (JBI) Critical Appraisal Tools for cross-sectional assessment of the risk of bias will be conducted by two independent independently by two authors (WM and PA) and discrepancies will be resolved through discussion with the third author (PW).

Strategy of data synthesis: The metaanalysis will be performed using the STATA program. Comparison between PA and EH, risk ratio (RR) with $95 \%$ confidence interval $(95 \% \mathrm{Cl})$, will be calculated to assess the effect size for outcomes which were binomial proportions. Standardized mean difference (SMD) with a $95 \% \mathrm{Cl}$ will be calculated for continuous outcome variables. Random-effect modelling will be performed in this study. The statistical significance level for this meta-analysis was set at $p<0.05$.

Subgroup analysis: Subgroup analysis will be also conducted to determine the effect of the potential confounders which will be categorized into sex, age, ethnicity, BMI, and demographic data matching.

\section{Sensitivity analysis: N/A}

Country(ies) involved: Thailand.

Keywords: primary aldosteronism, essential hypertension, diabetes mellitus, impaired fasting glucose, impaired glucose tolerance, insulin resistance.

Contributions of each author:

Author 1 - Worapaka Manosroi.

Email: worapaka.m@gmail.com

Author 2 - Pichitchai Atthakomol.

Email: p.atthakomol@gmail.com 